\title{
Perfluorinated Brønsted 'Superacids': Powerful Catalysts for the Preparation of Vitamin E
}

\author{
Thomas Netscher ${ }^{a \star}$, Werner Bonrath ${ }^{\mathrm{a}}$, Alois Haas ${ }^{\mathrm{b}}$, Eike Hoppmann ${ }^{\mathrm{b}}$, and Horst Pauling ${ }^{\mathrm{a}}$
}

\begin{abstract}
The key-step in the industrial synthesis of (all-rac)- $\alpha$-tocopherol (synthetic vitamin $E$ ) is the condensation reaction of trimethylhydroquinone with the $\mathrm{C}_{20}$ building block isophytol. For this Friedel-Crafts-type reaction, perfluorinated $\mathrm{NH}$ - and $\mathrm{CH}$-acidic compounds (perfluoroalkyl(aryl)sulfonyl imides and methides) have been applied as excellent catalysts, preferably in biphasic solvent systems.
\end{abstract}

Keywords: Biphasic catalysis · Friedel-Crafts alkylation · Perfluorinated $\mathrm{NH}$-acidic imides $\cdot \alpha$-Tocopherol · Tris(perfluoroalkylsulfonyl) methanes

\section{Introduction}

Vitamin $\mathrm{E}$ is the biologically most important fat-soluble antioxidant. The form of highest commercial value is synthetic (allrac)- $\alpha$-tocopherol (3), which is produced in a scale of over $25^{\prime} 000$ tons per year worldwide.

All industrial syntheses of (all-rac)- $\alpha-$ tocopherol (3) use the reaction of trimethylhydroquinone (1) with (all-rac)-isophytol (2) or a $\mathrm{C}_{20}$ equivalent thereof (Scheme 1). Several classical Lewis and Brønsted acids have been applied as catalysts in this reaction. Typical examples are zinc chloride and a mineral acid, $\mathrm{BF}_{3}, \mathrm{AlCl}_{3}, \mathrm{Fe} / \mathrm{HCl}$, or the combination of boric acid and carboxylic acids [1]. Such known catalysts, however, cause often serious corrosion and wastewater problems in large-scale production.

In order to avoid the disadvantages of the known syntheses of (all-rac)- $\alpha$-tocopherol (3), we focused our research on the

\footnotetext{
${ }^{*}$ Correspondence: Dr. T. Netscher ${ }^{\mathrm{a}}$

Tel.: +41616886755

Fax: +41616872201

E-Mail: thomas.netscher@dsm.com

aResearch and Development

DSM Nutritional Products

P.O. Box 3255

$\mathrm{CH}-4002$ Basel

(former company name: Roche Vitamins Ltd,

$\mathrm{CH}-4070$ Basel)

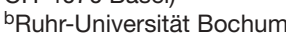

Fakultät für Chemie

D-44780 Bochum
}

development of more selective and environmentally friendly processes [2]. Polyfluorinated compounds were already involved in various approaches to accomplish this Friedel-Crafts-type alkylation reaction. Examples are scandium triflate [3] and the imide $\left(\mathrm{CF}_{3} \mathrm{SO}_{2}\right)_{2} \mathrm{NH}$ [4], as well as heterogeneous solid perfluoro-alkanesulfonic acids (i.e. their 'microencapsulated' variants and Nafion NR 50) [5].

\section{Synthesis of Catalysts}

In this communication we describe our findings when using fluorinated $\mathrm{NH}$-acidic catalysts of type $\mathbf{4}$ and $\mathrm{CH}$-acidic compounds $\mathbf{5}$ for the efficient synthesis of $\mathbf{3}$ from $\mathbf{1}$ and $\mathbf{2}$. Starting from commercially available sulfonyl halides (accessible by electrochemical fluorination), the highly air-sensitive bis[perfluoroalkyl(aryl)sulfonyl] imides $\mathbf{4}$ have been obtained via the sulfonamides and silyl derivatives (Scheme 2). Literature procedures [6-8] had to be adapted slightly to the individual compounds. The products were usually obtained after high-vacuum sublimation or short-path distillation from conc. $\mathrm{H}_{2} \mathrm{SO}_{4}$, or were isolated (e.g. aryl derivative $\mathbf{4 g}$ ) from the water-insoluble ammonium salt after use of cation exchange resin. Some of the

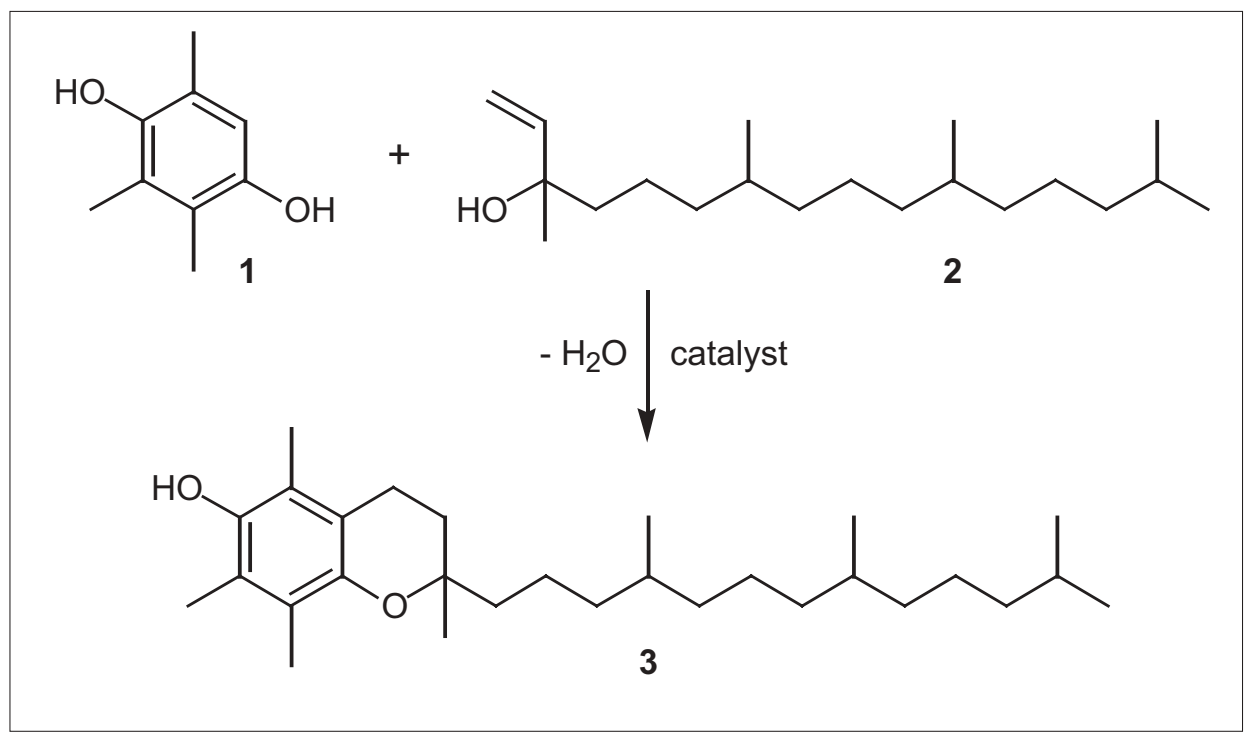

Scheme 1. Industrial synthesis of (all-rac)- $\alpha$-tocopherol (3) 


$$
\begin{aligned}
& \mathrm{R}_{\mathrm{f}}^{1} \mathrm{SO}_{2} \mathrm{X} \stackrel{\mathrm{NH}_{3}}{\longrightarrow} \mathrm{R}_{\mathrm{f}}{ }^{1} \mathrm{SO}_{2} \mathrm{NH}_{2} \stackrel{\text { 1) } \mathrm{MeONa}, \mathrm{MeOH}}{\text { 2) } \mathrm{HN}\left(\mathrm{SiMe}_{3}\right)_{2}} \\
& \mathrm{R}_{\mathrm{f}}{ }^{1} \mathrm{SO}_{2} \mathrm{~N}(\mathrm{Na}) \mathrm{SiMe}_{3} \stackrel{\text { 1) } \mathrm{R}_{\mathrm{f}}{ }^{2} \mathrm{SO}_{2} \mathrm{X}}{\text { 2) } \mathrm{H}_{2} \mathrm{SO}_{4} \text { conc. }} \underset{\mathrm{R}_{\mathrm{f}}{ }^{2}-\mathrm{SO}_{2}}{\stackrel{\mathrm{R}_{\mathrm{f}}{ }^{1}-\mathrm{SO}_{2}}{\mathrm{SH}}} \\
& \mathrm{X}=\mathrm{Cl}, \mathrm{F} \\
& \mathrm{R}_{\mathrm{f}}{ }^{1}, \mathrm{R}_{\mathrm{f}}{ }^{2}, \mathrm{R}_{\mathrm{f}}{ }^{3}=\text { perfluoroalkyl or pentafluorophenyl }
\end{aligned}
$$

Scheme 2. Preparation of perfluorinated imide catalysts 4

\begin{tabular}{|c|c|c|c|c|}
\hline 4 & $R_{f}^{1}$ & $\mathrm{R}_{\mathrm{f}}^{2}$ & Solvent system ${ }^{a}$ & Crude yield $\mathbf{3}[\%]^{\mathrm{b}}$ \\
\hline \multirow[t]{2}{*}{$\mathbf{a}$} & $\mathrm{CF}_{3}$ & $\mathrm{CF}_{3}$ & A & 89.5 \\
\hline & & & $\mathrm{B}$ & 91.0 \\
\hline \multirow[t]{2}{*}{ b } & $\mathrm{CF}_{3}$ & $\mathrm{C}_{4} \mathrm{~F}_{9}$ & $A$ & 89.6 \\
\hline & & & B & 94.0 \\
\hline c & $\mathrm{C}_{2} \mathrm{~F}_{5}$ & $\mathrm{C}_{4} \mathrm{~F}_{9}$ & $A$ & 90.2 \\
\hline \multirow[t]{3}{*}{ d } & $\mathrm{CF}_{3}$ & $\mathrm{C}_{6} \mathrm{~F}_{5}$ & $\mathrm{C}$ & 83.8 \\
\hline & & & $\mathrm{D}$ & 87.4 \\
\hline & & & $\mathrm{E}$ & 92.6 \\
\hline e & $\mathrm{C}_{2} \mathrm{~F}_{5}$ & $\mathrm{C}_{2} \mathrm{~F}_{5}$ & $B$ & 93.6 \\
\hline$f$ & $\mathrm{n} /$ iso- $\mathrm{C}_{3} \mathrm{~F}_{7}$ & $\mathrm{n} /$ iso- $\mathrm{C}_{3} \mathrm{~F}_{7}$ & B & 94.5 \\
\hline \multirow[t]{2}{*}{ g } & $\mathrm{C}_{6} \mathrm{~F}_{5}$ & $\mathrm{C}_{6} \mathrm{~F}_{5}$ & B & 87.2 \\
\hline & & & $E$ & 84.9 \\
\hline h & \multicolumn{2}{|c|}{$\mathrm{CF}_{2} \mathrm{CF}_{2} \mathrm{CF}_{2}$} & & В 94.5 \\
\hline i & $\mathrm{C}_{4} \mathrm{~F}_{9}$ & $\mathrm{C}_{4} \mathrm{~F}_{9}$ & $E$ & 94.0 \\
\hline k & $\mathrm{C}_{4} \mathrm{~F}_{9}$ & $\mathrm{C}_{6} \mathrm{~F}_{5}$ & $E$ & 93.3 \\
\hline m & $\mathrm{CF}_{3}$ & $\mathrm{C}_{8} \mathrm{~F}_{17}$ & $E$ & 86.4 \\
\hline $\mathbf{n}$ & $\mathrm{C}_{8} \mathrm{~F}_{17}$ & $\mathrm{C}_{8} \mathrm{~F}_{17}$ & $E$ & 85.6 \\
\hline o & $\mathrm{C}_{4} \mathrm{~F}_{9}$ & $\mathrm{C}_{8} \mathrm{~F}_{17}$ & $E$ & 91.1 \\
\hline
\end{tabular}

$$
\begin{aligned}
& \begin{array}{r}
\mathrm{R}_{\mathrm{f}}{ }^{1}-\underbrace{\mathrm{SO}_{2}}_{\mathrm{SO}_{2}} \mathrm{CH}_{2} \\
\mathrm{R}_{\mathrm{f}}{ }^{2}-\mathrm{SO}_{2}
\end{array} \\
& \text { 1) } \mathrm{LiC}\left(\mathrm{CH}_{3}\right)_{3} \text { or } \mathrm{CH}_{3} \mathrm{MgCl} \\
& \text { 2) } \mathrm{R}_{\mathrm{f}}^{3} \mathrm{SO}_{2} \mathrm{X} \quad \mathrm{R}_{\mathrm{f}}{ }^{1}-\mathrm{SO}_{2} \\
& \text { 3) } \mathrm{H}_{2} \mathrm{SO}_{4} \text { or } \mathrm{HCl} \\
& \text { 4) sublimation } \\
& \mathrm{X}=\mathrm{Cl}, \mathrm{F} \\
& R_{f}{ }^{1}, R_{f}{ }^{2}, R_{f}{ }^{3}=\text { perfluoroalkyl or pentafluorophenyl }
\end{aligned}
$$

\section{$\mathrm{R}_{\mathrm{f}}{ }^{3}-\mathrm{SO}_{2}$}

Table. Results of the condensation reaction of $\mathbf{1}$ and $\mathbf{2}$ in various solvents using $\mathrm{NH}$-acidic compounds of the type $\left(\mathrm{R}_{\mathrm{f}}^{1} \mathrm{SO}_{2}\right)\left(\mathrm{R}_{\mathrm{f}}{ }^{2} \mathrm{SO}_{2}\right) \mathrm{NH}(4)$

Scheme 3. Preparation of perfluorinated methide catalysts 5

imides 4 were commercially available [2]. Sulfonylation of bis(perfluoroalkylsulfonyl) methanes (Scheme 3) delivered the strongly acidic methides [tris(perfluoroalkylsulfonyl) methanes] 5 [9].

\section{Results of the Condensation Reactions}

Imides $\mathbf{4}$ as well as methides $\mathbf{5}$, used in really low catalyst amounts, yielded highly satisfying results in the condensation reaction. This applied to mildness of conditions, selectivity, and yield, which is remarkable when considering the mechanistic pathway of this transformation. In the preparation of $\alpha$-tocopherol (3) from $\mathbf{1}$ and $\mathbf{2}$ according to Scheme 1, the Friedel-Crafts alkylation reaction is followed by the chroman ring-closure step. It is of common knowledge that tertiary allylic alcohols like $\mathbf{2}$ easily dehydrate in the presence of acids [10] to form highly reactive carbocations. Therefore, the formation of considerable amounts of olefinic by-products is unavoidable in such reactions.

We were, however, pleased to find only small amounts (generally less than 4\%) of phytadienes (several $\mathrm{C}_{20}$-dienes) when $\mathbf{2}$ was reacted with $\mathbf{1}$ in the presence of catalytic amounts of perfluoroalkyl/aryl substituted imides 4 (0.1 mol\% based on 2$)$. The conversion of $\mathbf{2}$ was around $99 \%$, and yields of up to $94 \%$ (all-rac)- $\alpha$-tocopherol (3) were obtained (Table) [2][11]. Trissulfonyl methides $\mathbf{5}$ or selected metal salts thereof gave $\mathbf{3}$ in up to $96 \%$ yield [12]. In general, two-phase solvent systems like 
ethylene carbonate/heptane proved to be most effective.

In conclusion, perfluorinated $\mathrm{NH}$-acidic imides $\mathbf{4}$ and $\mathrm{CH}$-acidic methides $\mathbf{5}$ are excellent catalysts for the synthesis of (allrac)- $\alpha$-tocopherol (3) from trimethylhydroquinone (1) and isophytol (2). Best results were achieved by the application of biphasic solvent systems, i.e. combining the activity and selectivity of homogeneous catalysts with heterogeneous reaction media.

Received: January 8, 2004

[1] K.U. Baldenius, L. von dem BusscheHünnefeld, E. Hilgemann, P. Hoppe, R. Stürmer, in 'Ullmans Encylopedia of Industrial Chemistry', VCH Verlagsgesellschaft, Weinheim, 1996, pp. 478-488; T. Netscher, 'Synthesis and production of vitamin E', in 'Lipid Synthesis and Manufacture', Ed.: F.D. Gunstone, Sheffield Academic Press Ltd, Sheffield, UK, 1999, pp. 250-267.

[2] W. Bonrath, A. Haas, E. Hoppmann, Th. Netscher, H. Pauling, F. Schager, A. Wildermann, Adv. Synth. Catal. 2002, 344, 37.

[3] M. Matsui, N. Karibe, K. Hayashi, H. Yamamoto, Bull. Chem. Soc. Jpn. 1995, 68, 3569.

[4] K. Ishihara, M. Kubota, H. Yamamoto, Synlett 1996, 1045; M. Baak, W. Bonrath, H. Pauling (F. Hoffmann-La Roche), WO Patent Application 9821197, 1997 (Chem. Abstr. 1998, 129, 28106).

[5] F. Schager, W. Bonrath, Appl. Catal. A: General 2000, 202, 117; F. Schager, W. Bonrath, J. Catal. 1999, 182, 282; W. Bonrath (F. Hoffmann-La Roche), EP 782993 A1, 1996 (Chem. Abstr. 1997, 127, 109079).

[6] J.N. Meussdoerfer, H. Niederprüm, Chem. Ztg. 1972, 96, 582; H. Niederprüm, J.N. Meussdoerfer (Bayer AG), Deutsche Offenlegungsschrift 2239817, 1972 (Chem. Abstr. 1974, 80, 120260).

[7] J. Foropoulos Jr., D.D. Desmarteau, Inorg. Chem. 1984, 23, 3720; D.D. Desmarteau, M. Witz, J. Fluorine Chem. 1991, 52, 7.

[8] Alternative preparation via ammonium or metal salts: K. Pohmer, R. Weber, C. Dörzbach-Lange, K. Stachulla, H.-H. Moretto, M. Wienand (Bayer AG), DE Offenlegungsschrift 4217366 A1, 1992 (Chem. Abstr. 1994, 120, 301651); H. Sakaguchi, K. Fujii, S. Sakai, Y. Kobayashi, Y. Kita (Central Glass Co.), DE Offenlegungsschrift 19533711 A1, 1995 (Chem. Abstr. 1996, 124, 342658).

[9] L. Turowsky, K. Seppelt, Inorg. Chem. 1988, 27, 2135; A.G.M. Barrett, N. Bouloc, D.C. Braddock, D. Catterick, D. Chadwick, A.J.P. White, D.J. Williams, Tetrahedron 2002, 58, 3835; J. Nishikido, F. Yamamoto, H. Nakajima, Y. Mikami, Y. Matsumoto, K. Mikami, Synlett 1999, 1990.

[10] J. March, 'Advanced Organic Chemistry', $4^{\text {th }}$ Edition, John Wiley \& Sons, New York, 1992, p. 1011

[11] W. Bonrath, A. Haas, E. Hoppmann, H. Pauling, (Roche Vitamins), EP 1180517
A1, 2001 (Chem. Abstr. 2002, 136, 183967).

[12] W. Bonrath, A. Haas, E. Hoppmann, T. Netscher, H. Pauling (F. Hoffmann-La Roche), EP 1134218 A1, 2001 (Chem. Abstr. 2001, 135, 257368). 